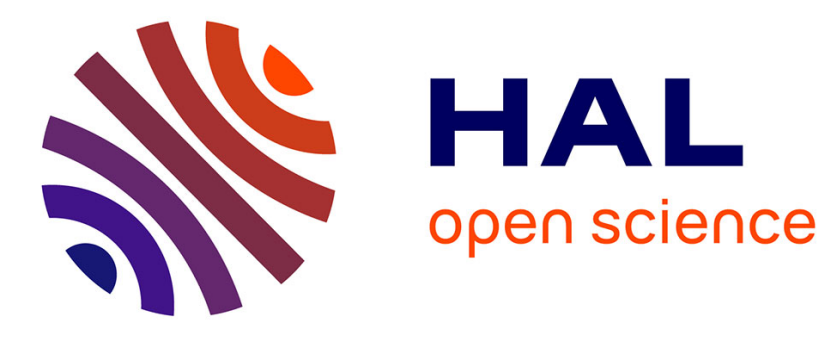

\title{
Actions hamiltoniennes de tores et jacobiennes généralisées
}

Michèle Audin

\section{To cite this version:}

Michèle Audin. Actions hamiltoniennes de tores et jacobiennes généralisées. Comptes rendus de l'Académie des sciences. Série I, Mathématique, 2002, 334 (1), pp.37-42. 10.1016/S1631073X(02)02197-0 . hal-00129661

\section{HAL Id: hal-00129661 \\ https://hal.science/hal-00129661}

Submitted on 8 Feb 2007

HAL is a multi-disciplinary open access archive for the deposit and dissemination of scientific research documents, whether they are published or not. The documents may come from teaching and research institutions in France or abroad, or from public or private research centers.
L'archive ouverte pluridisciplinaire HAL, est destinée au dépôt et à la diffusion de documents scientifiques de niveau recherche, publiés ou non, émanant des établissements d'enseignement et de recherche français ou étrangers, des laboratoires publics ou privés. 


\title{
ACTIONS HAMILTONIENNES DE TORES ET JACOBIENNES GÉNÉRALISÉES
}

\author{
par
}

\author{
Michèle Audin
}

\begin{abstract}
Résumé. Je décris la fibration en tores de Liouville d'un système intégrable sur les orbites coadjointes de $\mathrm{SU}(n)$ à l'aide de la jacobienne (relative) généralisée d'une famille de courbes singulières. Le système intégrable contient l'application moment de l'opération d'un tore maximal, ce sont ces hamiltoniens périodiques qui font apparaître la partie non compacte de la jacobienne.
\end{abstract}

Abstract. I describe the fibration in Liouville tori of an integrable system on the coadjoint orbits of $\mathrm{SU}(n)$ in terms of the (relative) generalized Jacobian of a family of singular curves. The integrable system contains the moment mapping of the maximal torus-these periodic Hamiltonians are responsible for the non compact part of the Jacobian.

\section{Abridged English version}

We consider a $2 n$-dimensional symplectic manifold endowed with an effective action of a $k$-torus $(k<n)$ and we assume that the momentum mapping

$$
\mu: W \longrightarrow \mathbf{R}^{k}
$$

can be completed into an intégrable system

$$
(h, \mu): W \longrightarrow \mathbf{R}^{n-k} \times \mathbf{R}^{k} .
$$

We assume moreover that the Hamiltonian system of one of the components of $(h, \mu)$ can be described by a Lax equation with a spectral parameter.

This is what happens in the case of the spherical pendulum, where $n=2, k=1$ and the symplectic manifold is the tangent bundle of the 2 -sphere

$$
W=\left\{(q, p) \in \mathbf{R}^{3} \times \mathbf{R}^{3} \mid\|q\|^{2}=1 \text { and } q \cdot p=0\right\} .
$$

The circle $S^{1}$ acts by rotations around the "vertical" axis. The momentum mapping is the periodic Hamiltonian

$$
\begin{aligned}
& K: T S^{2} \longrightarrow \mathbf{R} \\
&(q, p) \longmapsto \\
&(q \times p) \cdot e_{3},
\end{aligned}
$$

momentum with respect to the axis $e_{3}$. The function $H(q, p)=\frac{1}{2}\|q\|^{2}-q \cdot e_{3}$, total energy of the spherical pendulum, is constant on the orbits. and thus commutes with $K$. The Hamiltonian system associated with $H$ can be put in Lax form, but the spectral curve has genus 1, which is not enough to describe a two degree of freedom system. It is known however (see [1]) that a good model for the fibration in Liouville tori is the real part of a family of generalized Jacobians: the "good" spectral curve is singular.

The point here is that the $S^{1}$-action complexifies into a $\mathbf{C}^{\star}$-action, a fact that forces to use non compact Jacobians.

In this note, we will consider the (generic) coadjoint orbits $W$ of a compact semi-simple Lie group $G$, endowed with the action of a maximal torus $H$. The momentum mapping is simply the projection

$$
\mu: W \subset \mathfrak{g}^{\star} \longrightarrow \mathfrak{h}^{\star} .
$$


Fix a generic element $a \in \mathfrak{h}^{\star}$. Let $f$ be any $\operatorname{Ad}^{\star}$-invariant function on $\mathfrak{g}^{\star}$. It is known $[\mathbf{5}, \mathbf{2}]$ that the coefficients of the powers of $y$ in the $f(m+a y)$ 's form an integrable system on the Poisson manifold $\mathfrak{g}^{\star}$ and its coadjoint orbits.

For simplicity, consider the case where $G=\mathrm{SU}(n)$ and $H$ is the diagonal torus. The invariant functions can be grouped into the single polynomial

$$
P(x, y)=\operatorname{dét}(m+y a-x \mathrm{Id}) .
$$

The equation $P(x, y)=0$ defines a real affine curve in $C \subset \mathbf{C}^{2}$ and the coefficients of the polynomial are functions of $m$ in involution. Among them are the orbit invariants (obtained for $y=0$ ) and $n-1$ functions $F=\left(F_{1}, \ldots, F_{n-1}\right)$ that are linear in the diagonal entries of $m$, such that $F$ is the composition

$$
F: W \stackrel{\mu}{\longrightarrow} \mathbf{R}^{n-1} \stackrel{A}{\longrightarrow} \mathbf{R}^{n-1}
$$

of the momentum mapping of the torus action with a linear isomorphism. The other first integrals are the coefficients of $P$, higher degree functions of the entries of $m$. To fix values of these coefficients amounts to fix a specific curve $C$ and a common level set of the first integrals, that we denote $\mathcal{T}_{C}$.

Assume the affine curve $C$ to be smooth. We consider also its non singular completion $\widetilde{C}$ and its natural completion $\bar{C}$ in $\mathbf{P}^{1} \times \mathbf{P}^{1}$. We show that the curve $\bar{C}$ has a unique singular point at which $n$ distinct smooth branches intersect, while the smooth curve $\widetilde{C}$ has genus $g=\frac{(n-1)(n-2)}{2}$ (Proposition 2.2). We then consider the eigenvector mapping

$$
\varphi_{C}: \mathcal{T}_{C} \longrightarrow \operatorname{Pic}(\widetilde{C}),
$$

which associates, to the matrix $m$, the sub-bundle of $\widetilde{C} \times \mathbf{C}^{n}$ whose fiber at $(x, y)$ is the eigenspace of $m+y a$ for the eigenvalue $x$. We also consider the generalized Jacobian $\operatorname{Pic}(\bar{C})$ of the singular curve $\bar{C}$ (see [3]), we have a group morphism $\operatorname{Pic}(\bar{C}) \rightarrow \operatorname{Pic}(\widetilde{C})$ induced by the normalization $\widetilde{C} \rightarrow \bar{C}$, that is also a fibration with fiber $\left(\mathbf{C}^{\star}\right)^{n-1}$.

The main result is the following (Théorème 2.3):

Theorem. If the affine curve $C$ is smooth, the corresponding level $\mathcal{T}_{C}$ of the first integrals is regular. The eigenvector mapping defines an embedding (of real algebraic varieties) $\mathcal{T}_{C} / H \rightarrow \operatorname{Pic}(\widetilde{C})$, lifts into a (real algebraic) mapping $\mathcal{T}_{C} \rightarrow \operatorname{Pic} \bar{C}$, which is an an embedding, makes the diagram

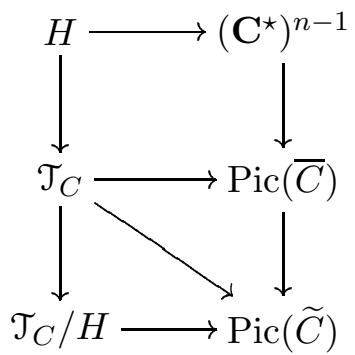

commute and includes the compact torus $H$ as the real part of $\left(\mathbf{C}^{\star}\right)^{n-1}$.

\section{Introduction}

L'objet de cette note est l'étude algébro-géométrique de systèmes intégrables dont une partie des intégrales premières sont des hamiltoniens périodiques. On considère une variété symplectique de dimension $2 n$, munie d'une opération (effective) d'un tore $T^{k}(k<n)$. On suppose que l'application moment

$$
\mu: W \longrightarrow \mathbf{R}^{k}
$$

se complète en un système intégrable

$$
(h, \mu): W \longrightarrow \mathbf{R}^{n-k} \times \mathbf{R}^{k}
$$

et que l'un des systèmes hamiltoniens (d'une composante de $(h, \mu)$ ) se décrit par une équation de Lax avec paramètre spectral. Avant d'en venir à l'objet principal de cette étude, le cas des orbites coadjointes de $\mathrm{SU}(n)$, commençons par un exemple très simple. 
Exemple 1.1. Ici $=2$ et $k=1$. La variété symplectique est le fibré tangent de la sphère $S^{2}$

$$
W=\left\{(q, p) \in \mathbf{R}^{3} \times \mathbf{R}^{3} \mid\|q\|^{2}=1 \text { et } q \cdot p=0\right\} .
$$

Le cercle $S^{1}$ opère par rotations autour d'un axe dit « vertical ». L'application moment est le hamiltonien périodique

$$
\begin{aligned}
& K: T S^{2} \longrightarrow \mathbf{R} \\
&(q, p) \longmapsto \\
&(q \wedge p) \cdot e_{3},
\end{aligned}
$$

moment par rapport à l'axe vertical $e_{3}$. La fonction $H(q, p)=\frac{1}{2}\|q\|^{2}-q \cdot e_{3}$ est constante sur les orbites de l'opération de $S^{1}$, donc commute avec $K$. C'est l'énergie totale du système mécanique dit « pendule sphérique ». Le système hamiltonien associé se met sous forme de Lax, la courbe spectrale est une courbe de genre 1, ce qui semble être trop peu pour un système à deux degrés de liberté. On sait (voir [1]) qu'en fait, le bon modèle pour la fibration en tores de Liouville est la partie réelle d'une famille de jacobiennes généralisées : la « bonne» courbe spectrale est singulière ${ }^{(1)}$.

La remarque que je vais systématiser dans cette note est le fait que l'action de $S^{1}$ se complexifie en une action de $\mathbf{C}^{\star}$, ce qui rend l'utilisation de «jacobiennes non compactes » et donc de courbes spectrales singulières naturelle et nécessaire.

\section{Orbites coadjointes de $\mathrm{SU}(n)$}

Les variétés symplectiques que je vais considérer sont les orbites coadjointes d'un groupe de Lie compact semi-simple, munies de l'opération coadjointe d'un tore maximal.

Soit donc $G$ un tel groupe de Lie, $W \subset \mathfrak{g}^{\star}$ une orbite coadjointe (munie de la structure symplectique de Kostant-Kirillov-Souriau), $H \subset G$ un tore maximal. L'opération coadjointe de $H$ sur $W$ est hamiltonienne, son application moment est simplement la projection

$$
\mu: W \subset \mathfrak{g}^{\star} \longrightarrow \mathfrak{h}^{\star} .
$$

On fixe un élément a générique de $\mathfrak{h}^{\star}$. Pour toute fonction $\mathrm{Ad}^{\star}$-invariante $f$ sur $\mathfrak{g}^{\star}$, on peut considérer $f(m+y a)$ (pour $m \in W$ et $y$ une variable indépendante) et le développer suivant les puissances de $y$, obtenant ainsi des fonctions sur $W$.

Proposition $2.1([\mathbf{5}, \mathbf{2}])$. Les coefficients du développement en y des $f(m+$ ay) quand $f$ parcourt les fonctions invariantes sur $\mathfrak{g}^{\star}$, forment un système intégrable sur la variété de Poisson $\mathfrak{g}^{\star}$ et ses orbites coadjointes.

Pour simplifier les énoncés, je vais me limiter au cas où $G=\mathrm{SU}(n)(n \geq 2)$. J'identifie comme il est d'usage l'algèbre de Lie $\mathfrak{s u}(n)$ (resp. son dual $\left.\mathfrak{s u}(n)^{\star}\right)$ à l'algèbre de Lie des matrices anti-hermitiennes (resp. des matrices hermitiennes) de trace nulle. On choisit pour $H$ le tore maximal des matrices diagonales dans $\mathrm{SU}(n)$. Ici $a$ est simplement une matrice diagonale de valeurs propres $a_{1}, \ldots, a_{n}$ réelles (de somme nulle). La dimension du groupe est $n^{2}-1$, celle d'une orbite générique $n(n-1)$, celle du tore maximal $n-1$. L'application moment associe à une matrice ses éléments diagonaux. On peut regrouper les fonctions invariantes en un polynôme à deux variables

$$
P(x, y)=\operatorname{dét}(m+y a-x \mathrm{Id}),
$$

l'équation $P(x, y)=0$ définissant une courbe affine réelle $C \subset \mathbf{C}^{2}$. La proposition 2.1 affirme alors que les coefficients de ce polynôme sont des fonctions (de $m$ ) en involution. Remarquons

- que $P(x, 0)=\operatorname{dét}(m-x \mathrm{Id})$, polynôme en $x$ dont les coefficients sont les invariants de l'orbite $W$ considérée,

- que la partie homogène de degré $n$ dans $P(x, y)$ est $\prod_{1 \leq i \leq n}\left(y a_{i}-x\right)$, de sorte que la courbe affine $C$ a $n$ points à l'infini en lesquels $\frac{x}{y} \sim a_{i}$

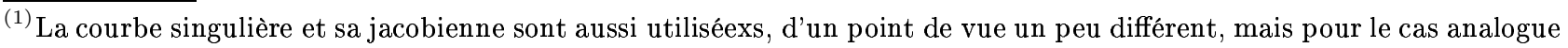
de la toupie symétrique, dans [4].
} 
- que la partie homogène de degré $n-1$ est

$$
\sum_{i=1}^{n} m_{i, i} \prod_{j \neq i}\left(y a_{j}-x\right)=y^{n-1} F_{n-1}+\cdots+y x^{n-2} F_{1},
$$

les $F_{i}$ étant, donc, des combinaisons linéaires des coefficients diagonaux de la matrice $m$. En d'autres termes, $F=\left(F_{1}, \ldots, F_{n-1}\right)$ est la composée

$$
F: W \stackrel{\mu}{\longrightarrow} \mathbf{R}^{n-1} \stackrel{A}{\longrightarrow} \mathbf{R}^{n-1}
$$

de $\mu$ et d'un isomorphisme linéaire.

Les autres coefficients de $P$ sont des fonctions au moins quadratiques des coefficients de $m$. Ce sont les autres intégrales premières. Donner des valeurs des coefficients revient à donner une courbe $C$ particulière et un niveau commun des intégrales premières. Je note donc $\mathcal{T}_{C}$ un tel niveau.

Supposons la courbe affine $C$ lisse. On considère aussi sa complétion non singulière $\widetilde{C}$ et sa complétion naturelle $\bar{C}$ dans $\mathbf{P}^{1} \times \mathbf{P}^{1}$. Ainsi, $\widetilde{C}$ est la normalisée de $\bar{C}$.

Proposition 2.2. Si $C$ est lisse, sa complétion non singulière $\widetilde{C}$ est une courbe de genre

$$
g=\frac{(n-1)(n-2)}{2} .
$$

La complétée $\bar{C}$ dans $\mathbf{P}^{1} \times \mathbf{P}^{1}$ a un unique point singulier, en lequel $n$ branches lisses se rencontrent transversalement.

Considérons maintenant l'application de vecteurs propres

$$
\varphi_{C}: \mathcal{T}_{C} \longrightarrow \operatorname{Pic}(\widetilde{C}) \text {. }
$$

Rappelons qu'elle associe à la matrice $m$ le (dual du) sous-fibré de $\widetilde{C} \times \mathbf{C}^{n}$ dont la fibre en $(x, y)$ est le sous-espace propre de $m+y a$ pour la valeur propre $x$. Cette application est bien définie dès que la courbe $C$ est lisse.

On considère aussi la jacobienne généralisée Pic $(\bar{C})$ de la courbe $\bar{C}$ (voir [3]). Rappelons que l'homomorphisme de groupes $\operatorname{Pic}(\bar{C}) \rightarrow \operatorname{Pic}(\widetilde{C})$ induit par la normalisation $\widetilde{C} \rightarrow \bar{C}$ est (aussi) une fibration de fibre $\left(\mathbf{C}^{\star}\right)^{n-1}$.

Théorème 2.3. Si la courbe affine $C$ est lisse, le niveau $\mathcal{T}_{C}$ correspondant est régulier. L'application de vecteurs propres induit un plongement (de variétés algébriques réelles) $\mathcal{T}_{C} / H \rightarrow \operatorname{Pic}(\widetilde{C})$, se relève en une application (algébrique, réelle) $\mathcal{T}_{C} \rightarrow \mathrm{Pic} \bar{C}$ qui est un plongement, fait commuter le diagramme

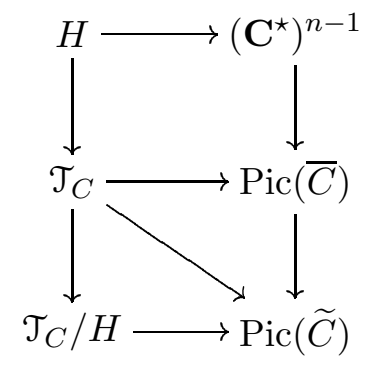

et inclut le tore compact $H$ comme la partie réelle de $\left(\mathbf{C}^{\star}\right)^{n-1}$.

Idées des démonstrations. La complétion naturelle de $C$ dans $\mathbf{P}^{2}(\mathbf{C})$ est lisse. On en déduit le genre de $\widetilde{C}$. La courbe $\bar{C}$ a un unique point singulier, pour $(x, y)=(\infty, \infty)$, où les $n$ asymptotes $y a_{i}-x=0$ se rencontrent. Ce point correspond à $n$ points $A_{1}, \ldots, A_{n}$ de $\widetilde{C}$. Le fibré des vecteurs propres a $n$ sections naturelles en les points $A_{1}, \ldots, A_{n}$, qui sont les vecteurs propres de la matrice diagonale $a$, vecteurs de la base canonique. C'est ce qui définit le relèvement de $\varphi_{C}$.

Les champs fondamentaux de l'action du tore $H$ sont envoyés, par l'application tangente à l'application de vecteurs propres, sur des éléments indépendants de l'espace tangent de $\operatorname{Pic}(\bar{C})$ (nuls dans celui de $\operatorname{Pic}(\widetilde{C})$.

Pour ce qui se passe en bas, on utilise une méthode classique en théorie des systèmes intégrables (voir par exemple [6]) qui permet de déterminer l'image de $\varphi_{C}$ dans $\operatorname{Pic}(\widetilde{C})$ (ce sont les classes des 
diviseurs $E$ de degré $g+n-1$ vérifiant $\left.h^{0}\left(E-A_{1}-\cdots-A_{n}\right)=0\right)$ et l'image inverse d'un élément de cette image.

Remerciements. Je remercie Nguyen Tien Zung de m'avoir fait découvrir le système intégrable dont il est question ici.

\section{Références}

[1] M. AUdin - « Hamiltonian monodromy via Picard-Lefschetz theory », preprint soumis (2001).

[2] A. V. Bolsinov - «Commutative families of functions related to consistent Poisson brackets », Acta Appl. Math. 24 (1991), p. 253-274.

[3] J. D. FAY - Theta functions on Riemann surfaces, Springer, Berlin, 1973, Lecture Notes in Mathematics, Vol. 352.

[4] L. Gavrilov \& A. Zhivkov - « The complex geometry of the Lagrange top », Enseign. Math. (2) 44 (1998), p. 133-170.

[5] A. S. Mishchenko \& A. T. Fomenko - « On the characteristic Euler equations in the dynamics of a $n$-dimensional solid body », Izv. Akad. Nauk SSSR 42 (1978), p. 369-415.

[6] A. G. Reiman - «Integrable Hamiltonian systems connected with graded Lie algebras », J. Soviet Math. 19 (1982), p. 1507-1545.

Version du 5 octobre 2001

Michèle Audin, Institut de Recherche Mathématique Avancée, Université Louis Pasteur et CNRS, 7 rue René Descartes, 67084 Strasbourg cedex, France • E-mail : Michele.Audin@math.u-strasbg.fr

Url: http://www-irma.u-strasbg.fr/ maudin 